Method. A comprehensive search was performed on PubMed, MEDLINE and PsycINFO, with identified studies screened against a priori inclusion/exclusion criteria. Eligible studies underwent quality-assessment using the Joanna Briggs Institute Critical Appraisal Tools, and data were synthesised qualitatively. Result. 17 publications were included, consisting of data on participants with obesity, binge eating disorder (BED), bulimia nervosa $(\mathrm{BN})$, anorexia nervosa ( $\mathrm{AN})$, and/or subclinical presentations. Studies were small (average sample size $\mathrm{n}=46.4$ ), predominantly female (87.9\%), and were highly variable in methodology, with different diagnostic tools, self-report measures, and emotional tasks/paradigms used.

Conclusion. The evidence suggests that HRV is a valid, objective biomarker of ER impairments in $\mathrm{AN}, \mathrm{BN}, \mathrm{BED}$, emotional eating, and obesity. Despite some inconsistencies, likely attributable to methodological heterogeneity, EDs/obesity appear to be characterised by irregular resting state vagal activity and abnormal stress reactivity. Furthermore, the autonomic dysfunction observed across EDs/obesity may be reversible by novel effective interventions such as HRV-biofeedback or PlayMancer videogame therapy.

\section{Vitamin D deficiency in a high secure forensic psychiatry hospital: A clinical audit and service evaluation}

George Gillett ${ }^{1 \star}$, Samantha Chudleigh-Warren ${ }^{2}$, Benedict Hardy ${ }^{3}$ and Charles Robert Gordon ${ }^{4}$

${ }^{1}$ IoPNN King's College London; ${ }^{2}$ Specialty Doctor in Psychiatry, Broadmoor Hospital; ${ }^{3}$ Consultant Forensic Psychiatrist, Broadmoor Hospital and ${ }^{4}$ Specialty Doctor in Psychiatry, Broadmoor Hospital ${ }^{*}$ Corresponding author.

doi: 10.1192/bjo.2021.123

Aims. To assess concordance with guidelines on monitoring vitamin D levels and prescribing prophylaxis or replacement. To assess the association between the implementation of local guidelines and prevalence of vitamin $\mathrm{D}$ deficiency.

Background. Vitamin D deficiency is associated with various adverse health outcomes including osteoporosis, fractures and myalgia. Most recently, vitamin D deficiency has been hypothesised as a risk factor for severe COVID-19 infection. Risk factors for vitamin D deficiency include incarceration, ethnicity, diet and a diagnosis of psychiatric disorder. Vitamin D deficiency is known to be prevalent among individuals within forensic mental health institutions.

Local Trust guidelines advise that vitamin D levels should be checked within one-month of hospital admission, followed by checks at three-monthly intervals. Recommendations for prescribing depend on patients' vitamin D levels; deficient $(<25 \mathrm{nmol} / \mathrm{L})$, insufficient $(25<50 \mathrm{nmol} / \mathrm{L})$ or adequate $(50-150 \mathrm{nmol} / \mathrm{L})$. We assessed concordance with these guidelines at Broadmoor Hospital, UK.

Method. Medical records, laboratory results and drug charts were assessed for a total of 75 patients across 15 wards. Data were collected using a standardised audit tool, including; date of admission, admission vitamin $\mathrm{D}$ level, most recent vitamin $\mathrm{D}$ level and the dose and frequency of vitamin $\mathrm{D}$ prescribed.

Result. $76.4 \%$ of patients had their vitamin D levels checked within one month of admission. $66.7 \%$ of patients had their vitamin $\mathrm{D}$ checked within the last 3 months. For patients with an admission vitamin $\mathrm{D}$ level recorded, $43.6 \%$ had deficient vitamin D levels, $43.6 \%$ had insufficient levels and $12.7 \%$ had adequate levels. For patients with a more recent serum vitamin D level, $14.5 \%$ had deficient levels, 38.7\% had insufficient levels and $46.8 \%$ had adequate levels. For patients with a documented serum vitamin D level, $21.4 \%$ were prescribed the correct dose, $22.9 \%$ were under-dosed, $14.3 \%$ were over-dosed and $41.4 \%$ received no dose where guidelines suggested they should.

Conclusion. Comparison of admission and most recent vitamin $\mathrm{D}$ levels suggests a general improvement in prevalence of vitamin $\mathrm{D}$ deficiency associated with the implementation of local guidelines. However, we identify significant areas for improvement. A substantial proportion of patients lacked admission or regular monitoring of vitamin D levels and a substantial proportion of patients were under-dosed or received no dose where guidelines suggested they should have. We propose that better concordance with guidelines may improve clinical outcomes further. This may prove especially important during the COVID-19 pandemic, given a potential association between vitamin $\mathrm{D}$ deficiency and severity of respiratory infection.

\section{HappyMaps: a single hub of resources on children and young people's mental health for parents and professionals}

\section{Georgina Griffiths ${ }^{1 \star}$, Jasmin Krischer ${ }^{2}$, Cara Roberts-Collins ${ }^{3}$ and Elaine Lunts ${ }^{2}$ \\ ${ }^{1}$ Avon and Wiltshire Partnership Trust; ${ }^{2}$ General practitioner and \\ ${ }^{3}$ Royal United Hospitals Bath NHS Foundation Trust \\ ${ }^{*}$ Corresponding author.}

doi: 10.1192/bjo.2021.125

Aims. Mental health issues in children and young people are a growing concern and the benefits of intervening early are well established for many mental health problems, but existing Child and Adolescent Mental Health Services (CAMHS) are often overstretched with variable waiting times for assessment. Many children also have problems which do not reach the referral thresholds and parents are left to find advice elsewhere. Existing resources for parents are scattered across many different websites and therefore difficult to access both for parents and professionals working with young people. With this in mind, and in consultation with CAMHS Bristol and many other stake-holders (including parents themselves) we designed an easily navigable website intended as a single comprehensive portal of resources for parents of children with mental illness and difficulties.

Method. Qualitative research methods were used to gather information about how the website should be designed and also to gather feedback once the website was live. Focus groups were performed with parents/carers and stakeholder discussions took place to inform the design of the website. Once the website was live, surveys via a Survey Monkey link on the website and Google Analytics were used to evaluate the website.

Result. 60,000 users have utilised the website since the launch in March 2019. Two thirds of users are women and one third are men. Most popular webpages that are visited are primary, secondary, help-in -a-crisis and self-help for young people. Positive feedback has been collected from both parents/carers and service providers. The website has continued to develop and is now a registered charity and has received community lottery funding, which will allow for further evaluation and developments.

Conclusion. HappyMaps has been successful in providing a single hub of information for parents/carers, GPs, CAMHS workers and teachers. Future work involves evaluating the website and attracting interest from other CAMHS teams and professionals in other areas of the UK so that they can create HappyMaps sections for their populations. 
Assessing the compliance of accurately documenting medication history in CAMHS - completion of the audit cycle

Laura Guest ${ }^{1 \star}$, Irangani Mudiyanselage ${ }^{2}$, Swetangi Ambekar ${ }^{2}$ and Sudheer Lankappa ${ }^{1}$

${ }^{1}$ Nottinghamshire Healthcare NHS Foundation Trust and

${ }^{2}$ Derbyshire Healthcare NHS FT

${ }^{\star}$ Corresponding author.

doi: 10.1192/bjo.2021.126

Aims. To assess the documentation of medication across all Child and Adolescent Mental Health Service (CAMHS) teams in the south region of Derbyshire Healthcare NHS Foundation Trust against a locally agreed protocol. The aim is to ensure accurate and timely documentation of medication history in a standardised way to reduce the risk of medication errors.

Method. We randomly selected 78 patients across seven teams within CAMHS that were currently prescribed medication as of November 2020. We reviewed each patient to see if medication history had been recorded in the specified section of the trust's patient database PARIS. We then cross referenced this information with the patient notes, clinic letters and prescriptions to review accuracy of information in terms of recording of drug name, dose, frequency, and whether the medication was regular or as required. We compared the data to the results of a previous audit in 2017 which used the same methods.

Result. Of the 78 patients, $74 \%(n=58)$ had medication recorded in the correct section of PARIS compared to $13 \%$ in the 2017 audit. We found that compliance varied between different CAMHS teams ranging from $0 \%$ to $100 \%$. Of those with medication history recorded, $86 \%$ had all drug names listed correctly, $79 \%$ had all drugs listed at the correct dose, $71 \%$ had the correct frequency recorded and $81 \%$ had whether the medication was regular, or PRN recorded.

Conclusion. Although we have seen improvement in standardised documentation of medication history since 2017, it remains difficult to rely on this information being up to date and reliable. There was a wide range of compliance in documentation of medication history across different teams, possibly reflecting how effectively the teaching following the previous 2017 audit had been delivered to each team. We have completed more teaching for medical and non-medical prescribers across all localities to highlight the importance of timely and standardised documentation. This is particularly important in CAMHS where the prescribing of medication often remains the responsibility of secondary care, with clinicians regularly prescribing on behalf of colleagues from other teams. Our findings support the move within the Trust towards a system where medication can be both documented and electronically prescribed in the same place (System One).

\section{Validation of the internet addiction test (IAT) to} Sinhalese and assessment of internet addiction among school children in Sri Lanka

Kavinda Gunathillaka ${ }^{2}$, Chamara Wijesinghe ${ }^{1 *}$,

Trivon Gunasekera ${ }^{1}$, Bhagye Premathilake ${ }^{1}$, Asiri Rodrigo ${ }^{1}$, Arunasalam Pathmeswaran ${ }^{3}$ and Lalith Kuruppuarachchi ${ }^{1}$

${ }^{1}$ Department of Psychiatry, Faculty of Medicine, University of Kelaniya; ${ }^{2}$ University Psychiatry Unit, Colombo North Teaching Hopsital and ${ }^{3}$ Department of Public Health, Faculty of Medicine, University of Kelaniya

${ }^{\star}$ Corresponding author.

doi: 10.1192/bjo.2021.127
Aims. To translate Young's Internet Addiction Test (IAT) to Sinhalese and validate for use in a Sri Lankan population. Following validation of the questionnaire, to use the validated questionnaire to assess the prevalence of internet addiction in a school going population in the Western province of Sri Lanka and identify characteristics of those addicted to the internet.

Background. The internet is widely used across the world and in Sri Lanka. Though essential for everyday life there are many negative aspects of internet use. Addiction to the internet is one such problem and identified to exist among the general population and students in other countries. The most common tool used to measure internet addiction is Young's internet addiction test. The phenomena of internet addiction has not been scientifically studied in Sri Lanka according to our knowledge.

Method. A school-based cross-sectional analytical study conducted in two stages among students aged 15 to 19 years. In stage 1 of the study, 200 students were administered the Sinhalese translation of the IAT and internal consistency and test retest validity assessed. Once validation of the scale was established the translated scale was used on a sample of 2800 students to assess presence of internet addiction.

Result. The Sinhala translation of the Internet addiction test showed good reliability and validity The Chrohnbach's alpha value was 0.78 and Pearson correlation coefficient of 0.85 and therefore suitable to use in a Sinhalese speaking population in Sri Lanka.

Internet addiction was identified among the study population. $8 \%$ of the entire study sample and $12.6 \%$ among those using the internet showed features of internet addiction. The majority of cases of internet addiction identified were mild $8.2 \%$ followed by moderate internet addiction in $3.6 \%$ and only $0.9 \%$ having severe internet addiction. There were no significant demographic or internet use related features identified among those with internet users and those not addicted to the internet.

Conclusion. This study demonstrated that the Sinhala translation of Young's IAT is suitable to assess internet addiction in Sri Lanka. It also identified that there are students in Sri Lanka who are addicted to the internet. This will possibly impact negatively on their lives at a crucial stage of development and have immediate as well as long term detrimental effects. More studies are required to identify characteristics of those who are addicted to the internet and to plan interventions.

\section{Differential attainment in undergraduate medical education: a systematic review}

Abhishek Gupta ${ }^{1 \star}$, Shreya Varma ${ }^{1}$, Radhika Gulati ${ }^{2}$, Natasha Rishi ${ }^{3}$, Nagina Khan ${ }^{4}$, Rohit Shankar ${ }^{5}$ and Subodh Dave ${ }^{6}$

${ }^{1}$ University of Birmingham, Medical School; ${ }^{2}$ Queen's University Belfast; ${ }^{3}$ South London and Maudsley NHS Foundation Trust; ${ }^{4}$ Touro University Nevada; ${ }^{5}$ Exeter Medical School, Cornwall Partnership NHS Foundation Trust, Chair Royal College of Psychiatrists South West Division and ${ }^{6}$ University of Bolton, Consultant Psychiatrist, Derbyshire Healthcare Foundation Trust; Hon. Asso. Professor, University of Nottingham, Dean Elect, Royal College of Psychiatrists ${ }^{*}$ Corresponding author.

doi: 10.1192/bjo.2021.128

Aims. Differential attainment (DA) amongst Black and Minority Ethnic (BAME) medical students and postgraduate trainees including Psychiatry trainees has been extensively documented in medical education, with non-white medical students being 2.5 times more likely to fail high-stake examinations compared to their White counterparts. The Equality Act 2010 places a 